

\title{
Model Based Capability Assessment of an Automated Eddy Current Inspection Procedure on Flat Surfaces
}

This document has been downloaded from Chalmers Publication Library (CPL). It is the author's version of a work that was accepted for publication in:

Research in nondestructive evaluation (ISSN: 0934-9847)

Citation for the published paper:

Rosell, A. ; Persson, G. (2013) "Model Based Capability Assessment of an Automated Eddy Current Inspection Procedure on Flat Surfaces". Research in nondestructive evaluation, vol. 24(3), pp. 154-176.

http://dx.doi.org/10.1080/09349847.2013.779401

Downloaded from: http://publications.lib.chalmers.se/publication/181434

Notice: Changes introduced as a result of publishing processes such as copy-editing and formatting may not be reflected in this document. For a definitive version of this work, please refer to the published source. Please note that access to the published version might require a subscription. 


\section{Model Based Capability Assessment of an Automated Eddy Cur- rent Inspection Procedure on Flat Surfaces}

Anders Rosell ${ }^{1}$, Gert Persson ${ }^{2}$

${ }^{1}$ GKN Aerospace Engine Systems, Trollhättan, Sweden, anders.ar.rosell@gknaerospace.com

${ }^{2}$ Chalmers University of Technology, Göteborg, Sweden

ABSTRACT: The probability of detection (POD) of a well controlled, automated eddy current procedure is evaluated in a numerical model and compared with experiments. The procedure is applied in laboratory environment with a single absolute probe which is positioned for raster scan over flat surfaces containing fatigue cracks. The variability of the signal amplitude, due to small fatigue cracks in the Titanium alloy Ti-6 Al-4V, is expected to mainly originate from crack characteristics and the index distance of the raster scan. The POD model is based on the signal versus crack size ( $\hat{a}$ versus $a)$ result. The presented procedure provides a well defined basis for a comparison between a simulated and an experimentally based POD assessment. Finite element analysis is used to model the eddy current method. A simplified fatigue crack model is first introduced and evaluated experimentally. Numerical computations are then used to build the corresponding model based POD curve which show good agreement with the experimental result. The model based POD curve is generated both by means of a parametric and a non-parametric approach. Differences between model based and experimental POD are discussed as well as the delta POD approach using transfer functions.

Keywords: Eddy current, Probability of detection (POD), Model based POD

\section{Introduction}

It is important to optimize maintenance and overhaul in order to accomplish light weight, cost efficient and reliable aero engines. The capability of inspection methods driven towards the detection of smaller and smaller defects is evaluated by probability of detection (POD) assessments within the aerospace industry. The objective of a POD assessment is to acquire a representative estimation of the capability 
of a non-destructive evaluation (NDE) procedure. It is of high importance that the reliability and performance of the NDE techniques used for flaw detection are validated and that the capability of detection of various flaws, such as cracks, is quantified and well understood.

In this work an automated eddy current (EC) procedure is considered. The framework for a POD assessment of such a NDE system is described in [1]. The procedures require extensive experimental work and a large number of cracks in order to show a valid POD estimate. This is often time consuming, costly and may require iterations of procedure parameter settings in order to arrive at the desired capability result. The experimental assessments are also often approximate as a consequence of the high costs involved to retrieve relevant defects in complex parts. Small variations in the procedure can change the POD and a full understanding of the impact from various parameters is therefore desired.

In order to support and understand the experimental work and capability estimations there is a need for modelling tools. Such tools can give efficient POD estimations at an early stage as well as an understanding of critical parameters and defect characteristics related to the inspection procedure. The possibility to model eddy current NDE has been developed over the last decades and the use of such models have been identified and used for POD estimation [2-7]. The approach in [6] was to include the uncertainty in the model based signal response from experimental data. The results were compared to an automated procedure but the authors concluded that the uncertainty of input parameters needs to be accounted for in the model based approach including the variation in crack characteristics. In [7] the authors used defined uncertainties of the input parameters to the model comparing the results to a manual eddy current inspection. This propagation of uncertainties through the mathematical model was described also in [5] but included in [7] was also a variability in flaw characteristics. This approach is used within this work which also includes a thorough investigation of the flaw description. The results are compared to an automated procedure which is described in detail and used for estimation of the uncertainties in input parameters. The comparison between model based and experimental results is here carried out for two different sets of procedure settings which enable to study the results both using full model assisted POD but also by the use of transfer functions. Two different approaches for model based POD are tested within this work, the first using the resulting data in a parametric POD model and the second not making any assumptions on the shape of the final POD curve. The modelling method used here is based on the finite element method. This method has previously been used for EC signal response predictions from realistic defects, such as tightly closed fatigue cracks [8].

A comparison between an experimental and a model based POD estimation is carried out within this 
work. The experimental procedure is simplified in the sense that the human influence is ignored and only one eddy current probe is used. This eliminates the variations introduced by those two factors. Signal response variation for automated procedures arises mainly from crack and sensor characteristics in automated eddy current procedures [9]. The focus here is to apply the modelling method conservative not overestimating the method capability, and to use relevant but simplified description of the characteristics of the fatigue cracks which are used in the experimental POD study. The model based POD assessment requires a three dimensional problem description. This puts high demands on how to use the modelling techniques in order to arrive at an efficient computational method. The objective of this work is to evaluate the possibilities and approaches of model based POD for automated eddy current procedures. This is achieved by comparing modelled results to a well controlled experimental estimation of method capability. The result serves as a validation of models in a statistical framework. It should, however, be emphasized that no specialized measurements on the variability of process parameters have been conducted other than described in this paper.

\section{Procedure}

The eddy current NDE procedure considered here is automated. An absolute probe is used and moved over flat surface samples. The raster scan index distance $L_{\text {scan }}$ is $0.5 \mathrm{~mm}$, see Fig. 1 . The probe is an absolute single coil with an outer diameter of $1 \mathrm{~mm}$, an inner diameter of $0.75 \mathrm{~mm}$ and a length of $1 \mathrm{~mm}$, operating at a frequency of $1 \mathrm{MHz}$. The material is Ti-6Al-4V with a conductivity of $0.58 \mathrm{MS} / \mathrm{m}$ and the standard depth of penetration is thus $0.66 \mathrm{~mm}$. The signal amplification (gain) is equal in the $x$ and $y$ component of the impedance using an Elotest B1 commercial instrument. The impedance response is measured by the signal amplitude, not making any considerations about the phase. This approach is adopted since it is general but yet simplifies the comparison with modelled results. Measuring the impedance signal amplitude may give rise to false indications from lift-off variations. In this procedure the scan speed is low $(1 \mathrm{~mm} / \mathrm{s})$ and the probe is put in contact with the smooth surface using a spring loaded fixture reducing such unwanted variations. A protective PTFE tape is put on the sensor tip to avoid wear. The thickness of the tape is $0.1 \mathrm{~mm}$. A rectangular calibration notch with dimensions length $=0.76 \mathrm{~mm}$, depth $=0.38 \mathrm{~mm}$ and width of approximately $0.076 \mathrm{~mm}$ is used. The notch is placed in a thick block of Ti-6AL-4V with smooth surface similar to the test samples. The signal amplitude response $|Z|$ of a defect is always compared to the response of the calibration notch, denoted $\left|Z_{N}\right|$. For this simplified procedure only one probe and one calibration block are considered. The positioning of specimens may place the crack in various positions relative the scan plan. The cracks are aligned with the direction of the scan but may deviate slightly from this and it is assumed that the orientation is normally 
distributed with a mean of $0^{\circ}$ and a standard deviation of approximately $1-5^{\circ}$. The signal threshold is set to $20 \%$ of the calibration notch and the detection threshold i.e. noise level is approximately $25 \%$ of that, thus corresponding to $5 \%$ of the calibration notch signal amplitude response. The procedure parameters are summarized in Table 1.

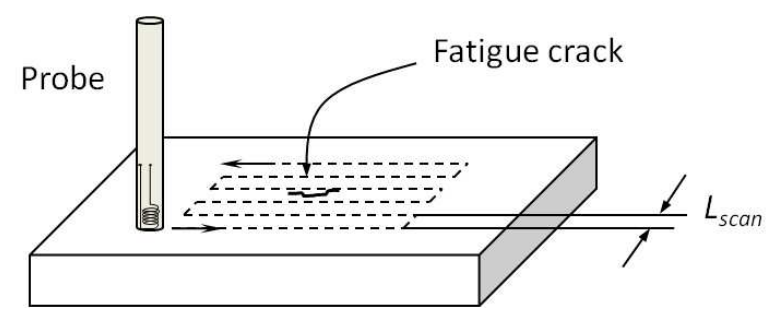

Fig. 1. Scan plan of eddy current procedure.

Table 1. Summary of eddy current procedure.

\begin{tabular}{ll}
\hline EC signal measure & Impedance amplitude relative calibration \\
Probe type & Absolute, air-core without shielding \\
Probe dimensions & $\mathrm{ID}=0.75, \mathrm{OD}=1, \mathrm{~L}=1[\mathrm{~mm}]$ \\
Frequency & $1 \mathrm{MHz}$ \\
Scan index distance & $L_{\text {scan }}=0.5 \mathrm{~mm}$ \\
Scan speed & $1 \mathrm{~mm} / \mathrm{s}$ \\
Inspection type & Automated on flat surface \\
Defects & Fatigue cracks aligned with scan direction \\
Material & Ti-6Al-4V \\
Material conductivity $\sigma_{0}$ & $0.58 \mathrm{MS} / \mathrm{m}$ \\
Calibration notch & Length $=0.76$, Depth=0.38, Width=0.076 [mm $]$ \\
Noise threshold $\hat{a}_{t h}$ & $5 \%$ \\
Decision threshold $\hat{a}_{d e c}$ & $20 \%$ \\
\hline
\end{tabular}

\section{Methods for experimental capability assessment}

\subsection{Experimental set up}

An eddy current measurement system, shown in Fig. 2a, was set up for the experimental procedure evaluation. The system is build up by two linear actuators connected to form a $x-y$ table. The probe is moved on the actuators by stepper motors connected to a motion system built up in LabVIEW. A measurement acquisition system is connected to the Elotest B1 instrument and the impedance of the probe is captured as an analogue signal together with the probe position with a sampling rate of $50 \mathrm{~Hz}$ during scan. The position accuracy is of the order of 1-10 $\mu \mathrm{m}$. The system allows detailed measurements of flat samples using a small scan index distance as presented in Fig. 2b. For such evaluations of signal responses as a function of $x$ and $y$ coordinates a scan index distance of $50 \mu \mathrm{m}$ is used. 


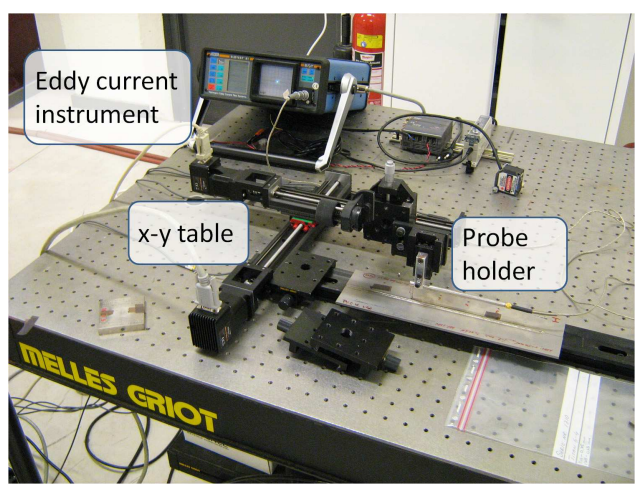

(a) System set up.



(b) Detailed scan over a fatigue crack.

Fig. 2. Experimental system.

\subsection{Significant variables}

The first step in order to be able to understand procedure variations consists of identifying the parameters which are possible sources of uncertainty in the NDE process. Once identified, a statistical description of each parameter must be carried out in order to understand the variability that is to be used in the models.

There are in general three kinds of influencing parameters in an eddy current procedure. First those associated with the handling and characteristics of the probe and equipment. Secondly the sample or component features and last the characteristics of the defect. An overview of the variables and how they are treated here is given in Table 2. A more complete description of all parameters that should be considered in a capability assessment for a general eddy current procedure is presented in [1] and in [10]. Some of the specified variables in Table 2 are stated quite simply such as the fixed bulk material property, which in the general case can vary due to for example thermal history, microstructure, manufacturing e.g. cast or forged. Variations in those properties can introduce differences in permeability and conductivity and thus contribute to variability in eddy current crack signal response and to the probability of false alarms (PFA). Here, such bulk material variations are considered to be small and are ignored. Variables connected to the human factor and interpretation of signals and measurements are not presented in the table.

Small surface variations due to roughness corresponding to variations in tilt angle are in this case considered to contribute as a variation in lift-off only, which is a valid assumption for small angles [11]. A lift-off curve may be calculated using an analytical model according to [12]. This model is used in order to characterize the lift-off of the measured signal. A signal from a scan during the measurements of 
Table 2. Variables considered in the automated eddy current procedure.

\begin{tabular}{l|ll} 
& Source of signal response variation & \\
\hline Test Frequency & Probe handling & Scan index distance \\
Probe & Lift-off \\
Gain & & Tilt angle \\
Signal evaluation & Scan speed \\
Threshold levels & & Repetition \\
Filters & & Positioning \\
Calibration notch & \\
Cables & Component & Surface roughness \\
\cline { 2 - 3 } $\begin{array}{ll}\text { Digitalization } \\
\text { Signal capture }\end{array}$ & Shape \\
\cline { 2 - 2 } Bulk material properties & \\
Temperature $\left(20^{\circ} \mathrm{C}\right)$ & & Width \\
Instrument & & Electric contacts \\
\cline { 2 - 2 } & & Orientation \\
\hline${ }^{a}$ May be considered in modelling aspects as a source of variation for a general procedure.
\end{tabular}

${ }^{a}$ May be considered in modelling aspects as a source of variation for a general procedure.

the experimental POD assessment is presented in Fig. 3a. The actual signal amplitude is retrieved as the signal without noise using moving average. The lift-off variation is considered from this signal using the analytical model. The signal amplitude is captured by the $Z_{x}$ and $Z_{y}$ impedance channels which both contain noise related to the electric circuits and lift-off. Here, the amplitude noise is characterized directly which leads to a simplified treatment. To evaluate the electric noise and the variation in lift-off, the measured signal is studied. The noise and lift-off characteristics are presented in Fig. 3b, together with the estimated standard deviations assuming a normal distribution in both cases.

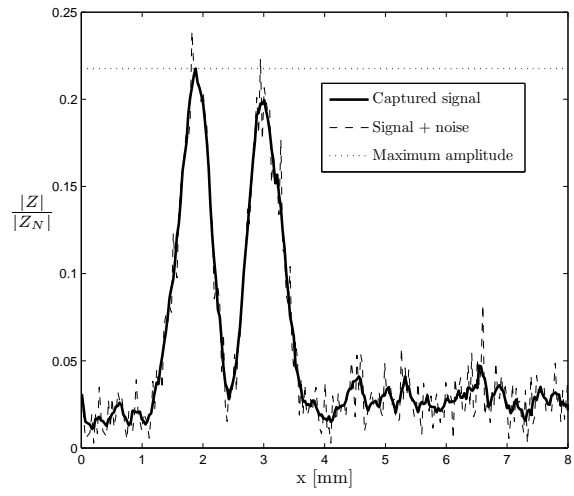

(a) Signal capture.
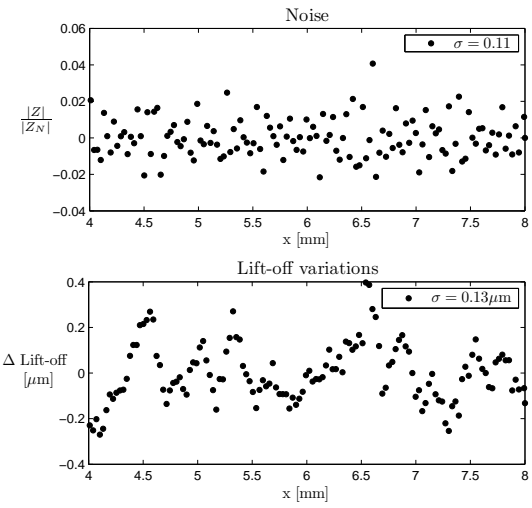

(b) Noise and lift-off variations.

Fig. 3. Signal processing in the eddy current measurement.

The repetition uncertainty and scan speed are not included as sources of variation. This is due to the experimental set up, which is optimized for position accuracy but results in low scanning speed. The sources of variations in Table 2 may for this procedure be significantly reduced and the important factors 
that are expected to affect the POD are in this procedure:

- Scan position relative to the crack, assumed to be uniformly distributed in the interval $[-0.25,0.25]$ $\mathrm{mm}$.

- Crack orientation relative to the scan direction, normally distributed with mean and standard deviation approximately $\mu_{\text {orient }}=0^{\circ}$ and $\sigma_{\text {orient }} 1-5^{\circ}$, respectively.

- Lift-off, assumed to be normally distributed with mean $\mu_{L}=0.1 \mathrm{~mm}$ and standard deviation $\sigma_{L}$ around $1 \mu \mathrm{m}$.

- Fatigue crack characteristics.

\subsection{Methods to estimate the experimental POD curve}

One of the most difficult types of defects to detect that also are one of the most severe to load carrying components, is the small and tightly closed fatigue crack [10]. Such cracks are possible to intentionally produce in test objects with a fatigue test and are recommended to use for capability estimation. The number of cracks should be at least 40 according to [1] and at least 30 according to [9] for an $\hat{a}$ versus $a$ analysis, where the signal response $\hat{a}$ is recorded for each crack of size $a .53$ cracks are used for the POD assessment within this work. All cracks are created with the same parameters in the fatigue process but are exposed to different numbers of cycles rendering the size variation. The sizes are measured on the sample surfaces and the half-circular geometry is verified by destructive evaluation of a few cracks. The cracks that were opened for size verification had sizes from 0.89 to $2 \mathrm{~mm}$ in length and had all a depth lying within $\pm 4 \%$ of half the surface length. The samples are ground for removal of the crack initiation notches. For the experimental POD curve estimation it is assumed that the signal response variation is constant for all crack sizes $a$. The relation between $\hat{a}$ and $a$ that is best describe as linear with constant variance at all crack sizes is selected, considering also $\log (\hat{a})$ versus $a, \hat{a} \operatorname{versus} \log (a)$ and $\log (\hat{a})$ versus $\log (a)$. The parametric POD curve estimation together with the $95 \%$ confidence limit is modelled following the steps in [1]. The derived result is representing a parametric POD model assuming that the curve follows a cumulative normal distribution function.

\section{Experimental results}

Aiming at normally distributed signal responses with a mean value at the linear regression line and constant variance for all crack sizes yields best fit with a $\log (\hat{a})$ versus $\log (a)$ relation. This decision can be aided by evaluations using for example Kolmogorov-Smirnov or Durbin-Watson statistical tests on the 
residuals. The resulting signal amplitudes and residuals for that case are presented in Fig. 4a together with the linear regression line. The corresponding $P O D(a)$ is presented in Fig. 4b together with the lower $95 \%$ confidence limit. The constant variance, estimated with maximum likelihood principles, of the data along the regression line is $v_{M L}=0.0394$. The resulting values of $a_{90}$ and $a_{90 / 95}$ are $0.8031 \mathrm{~mm}$ and $0.8313 \mathrm{~mm}$ respectively.

The variation of signal responses around the regression line is assumed to mainly originate from the variation in crack characteristics. A second data collection was therefore carried out with a doubled scan index distance, $L_{\text {scan }}$, of $1 \mathrm{~mm}$. The procedure is otherwise identical with the data in Table 1 . This modified procedure will reflect the change in POD related to the scan index variable as the same cracks are used for these experiments. The result is included in Fig. 4. The constant variance is $v_{M L}=0.0790$ and the resulting values of $a_{90}$ and $a_{90 / 95}$ are $0.9199 \mathrm{~mm}$ and $0.9594 \mathrm{~mm}$ respectively. It is clear from this data that the change in scan index distance has an impact on the resulting POD curve and the lower confidence limit. It should also be mentioned that these procedures are not intended to represent the optimum selection in order to achieve the best possible POD of a eddy current evaluation but rather be a typical example using a simple absolute air-core probe.
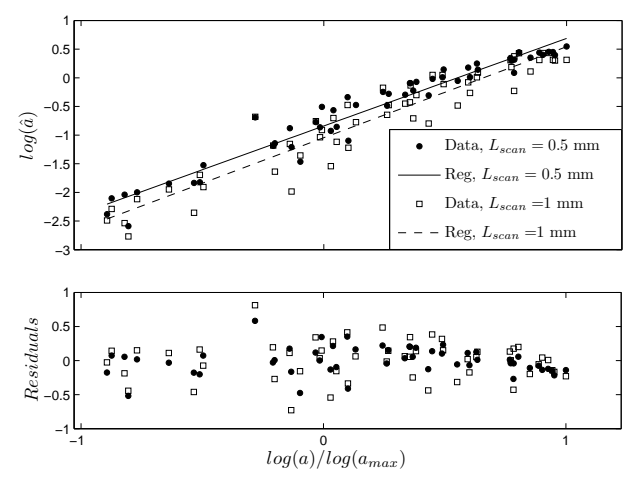

(a) Experimental $\hat{a}$ vs $a$ data.

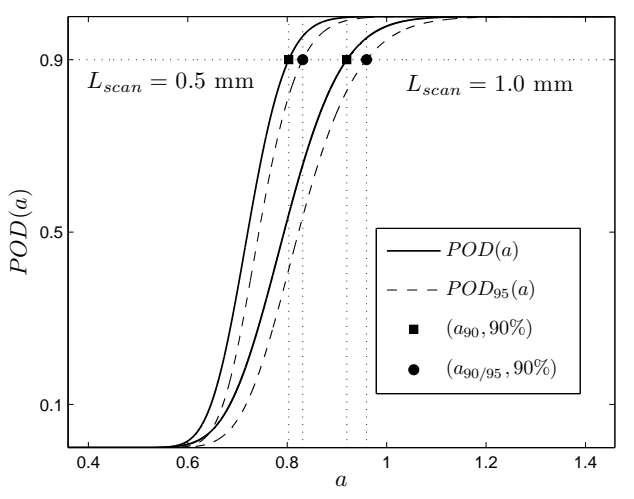

(b) Estimated POD curve.

Fig. 4. Results of experimental POD.

\section{$5 \quad$ Fatigue crack model}

Model assisted POD estimation must make use of a validated model that is able to include variations in the significant procedure variables. The numerical modelling method used is validated for the specific crack type, probe and material that are used within this work [8]. The cracks used here are tightly closed and electric connections between the crack surfaces are appearing. The state of the electrical contact is 
thus first evaluated in order to use a relevant fatigue crack model.

The method capability must be described using POD as different defects of the same size will produce different signal responses. This means that defects of the same size may sometimes be detected and sometimes not. This is related to the fact that inspection systems are driven to their extreme performance. One of the most important sources of this signal variation is the characteristics of the individual crack. Factors that may be considered in the fatigue crack characteristics are presented in Table 2 . It is however difficult to estimate the distribution of parameters such as shape, electric contact, angle to the surface and branching of fatigue cracks non-destructively. The fatigue cracks used here are considered to give variation in signal response mainly due to electric contacts between the crack faces. This could be modelled in combination with a variation in shape or angle in a more complex model if such data are available. The variable used must reflect the signal response of the crack and may depend on several factors such as material type and process used in the manufacturing of the cracks. The aim is to build a simplified model that reflects the relevant response characteristics at the different crack sizes. Such a model should be relevant in a statistical perspective but may deviate from the best model of an individual crack.

The characteristics of the fatigue crack are modelled with electrical connections represented by a non-zero conductivity tensor $\sigma_{i j}$ applied to the elements constituting the crack. The tensor is modified according to

$$
\sigma_{i j}=\left[\begin{array}{ccc}
\sigma_{x x}(r, \varphi) & 0 & 0 \\
0 & 0 & 0 \\
0 & 0 & 0
\end{array}\right]
$$

where the $x$-axis is directed normal to the crack surface. The model is three dimensional with the fatigue crack geometry approximated as a half-circular with a radius $r_{D}$ according to Fig. 5. The crack model used closes the crack in the radial direction according to

$$
\sigma_{x x}= \begin{cases}\sigma_{x x}(r, \varphi)=0 & r<(1-\zeta) \cdot r_{D} \\ \sigma_{x x}(r, \varphi)=\sigma_{c} & r \geq(1-\zeta) \cdot r_{D}\end{cases}
$$

A number of fatigue cracks were measured with a scan index distance of $50 \mu \mathrm{m}$. This was carried out in order to determine the maximum amplitude and to study the characteristics of the signal as a function of the spatial coordinates $x$ and $y$. The measurements were conducted in order to build a crack model that enables a relevant signal response for all crack sizes used. The crack model is also used to estimate, 


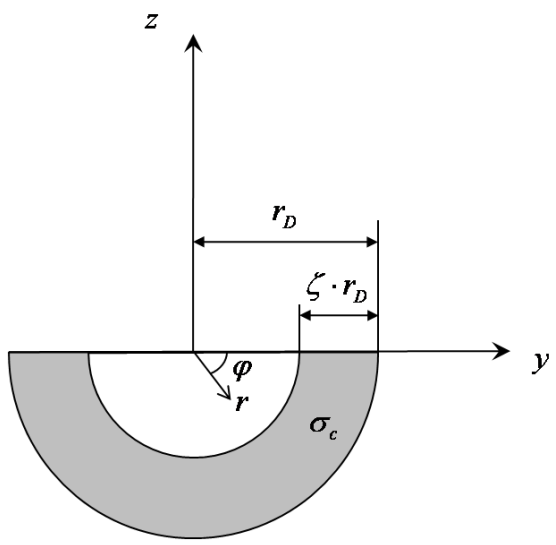

Fig. 5. Simplified fatigue crack model.

qualitatively, the bounds that are relevant for variation of the response. A number of values of $\zeta$ in Eq. (2) together with $\sigma_{c}=0.8 \cdot \sigma_{0}$ gives the maximum signal responses presented in Fig. 6 plotted together with the experimental data. The model was selected after evaluation of many different options and has two parameters, i.e. $\sigma_{c}$ and $\zeta$. Variations in signal responses can however be represented with a variation in only one of them, i.e. $\zeta$. The model has the benefit of being acceptable for all crack sizes considered both in nominal value and in variation of the signal response using the same parameters. This simplified model must be assumed to hold only for this particular procedure, concerning the bulk material and applied frequency of the magnetic field.

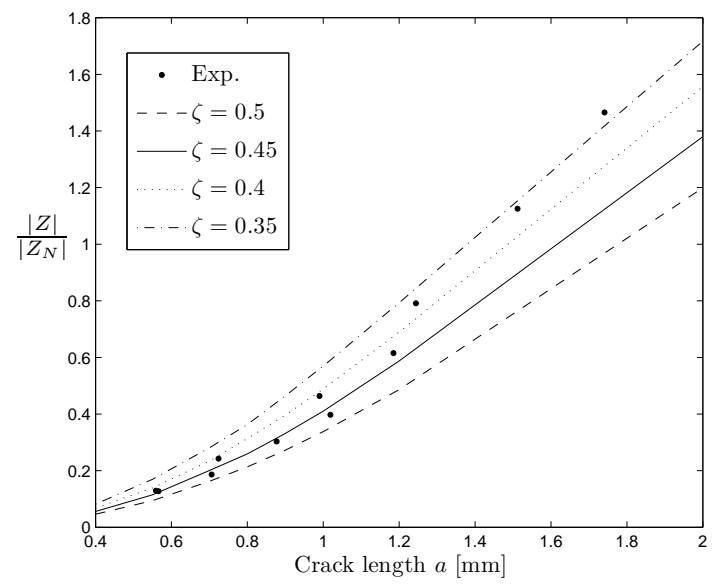

Fig. 6. Modelled signal response compared to experimental data.

Experimental measurements on the reference notch and the fatigue crack show smoothness and symmetric characteristics in the obtained signal. This has been an input for the suggested crack model. The characteristics can be concluded from Fig. $2 \mathrm{~b}$ where the amplitude is presented as a function of the 
spatial coordinates.

A crack width of $25 \mu \mathrm{m}$ is used in the model. This is representing approximately $3.8 \%$ of the depth of penetration which is $661 \mu \mathrm{m}$. The crack width effect on signal amplitude is previously studied by finite element models [8]. The response to closed cracks are evaluated more general in [13, 14] for various configurations and an analytical model for an ideal crack with negligible width is described and compared to experiments in [15]. The results in Table 3 and Fig. 6 show that the non-zero width used in this model cannot account for the reduced amplitudes of the fatigue cracks, therefore suggesting the electric contacts between the crack faces. The model that is used represents cracks of sizes around $1 \mathrm{~mm}$ well but is underestimating signal amplitude responses for larger cracks, which is clear from Fig. 6. This may indicate that the contact model should be modified for larger cracks. Predicting lower signal amplitude will, however, contribute to a conservative result and it is reasonable to use the same model for all cracks in order to describe variations in contact characteristics in a way that can be used for all crack sizes. One typical eddy current signal characteristic for small defects is that the amplitude has a maximum as the sensor is positioned at each corner of the crack and drops at a position over the centre. The contact model used here underestimates the spatial extension of cracks, which is concluded from the reduced distance between the amplitude peaks arising at the corners of the crack, see Table 3 . This shows the approximate nature of the model but will contribute as a conservative description of the cracks. The experimental POD is conducted using cracks aligned with the scan direction and the distance between the peaks is thus of less importance in that case. A comparison of the signal amplitudes in the case where crack contacts are ignored is also included in the table. Data from three of the cracks around the size of $a_{90 / 95}$ are specified.

Table 3. Crack model validation.

\begin{tabular}{|c|c|c|c|c|c|c|c|}
\hline \multirow[t]{2}{*}{ Crack } & \multirow[t]{2}{*}{ Size $[\mathrm{mm}]$} & \multicolumn{2}{|c|}{ Exp data } & \multicolumn{4}{|c|}{ Model data } \\
\hline & & $|Z| /\left|Z_{N}\right|$ & Peak dist. $[\mathrm{mm}]$ & $|Z| /\left|Z_{N}\right|$ & $\begin{array}{l}\sigma_{x x}=0 \\
\text { Peak dist. }[\mathrm{mm}]\end{array}$ & $\begin{array}{r}\sigma_{x x}-1 \\
|Z| /\left|Z_{N}\right|\end{array}$ & $\begin{array}{l}(2), \zeta=0.45 \\
\text { Peak dist. }[\mathrm{mm}]\end{array}$ \\
\hline$\overline{\mathrm{A}}$ & 0.724 & 0.2176 & 1.10 & 0.67 & 1.08 & 0.21 & 0.98 \\
\hline B & 0.99 & 0.4215 & 1.21 & 1.15 & 1.2 & 0.41 & 1 \\
\hline $\mathrm{C}$ & 1.185 & 0.6149 & 1.32 & 1.51 & 1.3 & 0.57 & 1.04 \\
\hline REF & 0.76 & 1 & 1.2 & 1 & 1.2 & - & - \\
\hline
\end{tabular}

\section{Model based POD}

Model assisted POD can have several approaches depending on the nature of the model and the nondestructive evaluation process studied. A delta POD or transfer function approach may be used if some parameters are studied in experiments and other in models. Another goal can be to build a full model 
based POD. Estimation of the POD in that case relies on the possibility to include all relevant variables and their distributions. This approach is considered here and can be divided in two different concepts based on the access and use of modelled data. The first concept for a model based POD estimation uses a limited number of signal response calculations resulting in an output analogous with the experimental case. The produced result will resemble the experimental $\hat{a}$ versus $a$ data and may be used similar to this for producing the parametric POD curve. A benefit of this approach may be that a lower confidence limit can be estimated with the same procedures as the experimental POD analysis and that the handling of data is analogous. The second concept requires a large number of calculations in order to estimate signal distributions received for individual crack sizes. In this work this is achieved by the use of a simplified meta-model based on a limited number of FEM computations. Monte Carlo computations are then carried out in order to make POD estimation, by calculating the fraction of signal responses that lies above the decision threshold. The first concept is generating a parametric POD curve estimate and the second a non-parametric result as no assumptions are made on the shape of the resulting POD curve.

\subsection{Approach 1: Model generated data for parametric POD}

The FEM computation is carried out using second order vector elements [8]. The volume in proximity to the defect is resolved using element sizes of approximately $10 \%$ of the penetration depth in order to describe the geometry and width of the defect, also giving a converged FEM solution. First an approximation on the spatial signal distribution is introduced in order to build efficient FEM computations. Small deviations in crack characteristics give a relative signal response as a function of $x$ and $y$ that is similar for all values of process parameters in the relevant interval. This allows usage of the signal response surface altered only by a scaling of the amplitude according to the process parameters, which in this case are contact and lift-off conditions. Using the amplitude response surface is convenient as scan orientation and position relative the defect can be sampled fast and efficient computations achieved. The maximum amplitude response must in general be evaluated considering multiple scans over the area containing the crack. Fig. 7 displays the signal amplitude as a function of sensor position $(x, y)$. The lines show the sensor positions during a scan and the maximum amplitude signal is detected along one of those paths, not in general the one closest to the centre of the defect. Making use of the approximation of constant signal amplitude distribution for each crack size will decrease the computation time considerably as all scan paths nearby the defect can be evaluated by calculation of one sampled configuration of lift-off and contact parameters. The approximation decreases the computation time in this case about a factor of 100. The approach needs an initial computation of several sensor positions each requiring a few minutes of computation time using a finite element model with approximately 100.000 degrees of freedom. Here, 
about 400 positions are calculated in the first quadrant resulting in a crack signal response according to Fig. 7 using symmetry. The distance between each sensor position is $50 \mu \mathrm{m}$ in order to resolve the signal during a scan over the defect within the model. This is carried out for each defect size selected for input to the POD curve. From this result various defect orientation- or location distributions can be evaluated.

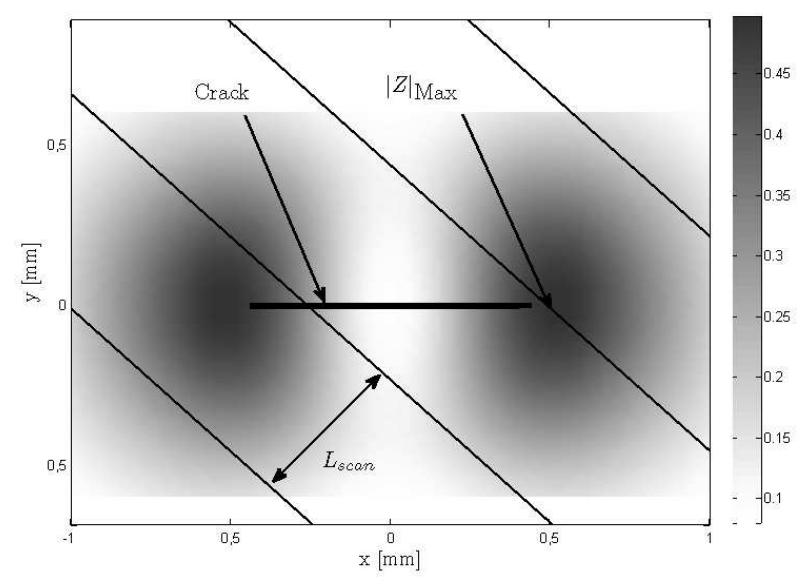

Fig. 7. Signal amplitude response as a function of sensor coordinates. Lines indicate sensor positions during a raster scan.

The assumption that the spatial distribution is constant for each crack size within the parameter interval gives an error in the order of a few percent. This is concluded by comparing the shape, for nominal and extreme values of the parameters, in areas where maximum signal amplitude may be detected. The nominal values for the contact condition parameter $\zeta$ and lift-off considered here are 0.45 and $0.1 \mathrm{~mm}$ respectively. The error as a function of sensor position around a $0.75 \mathrm{~mm}$ long crack is presented in Fig. 8 using $\zeta=0.5$ and a lift-off of $0.11 \mathrm{~mm}$. The approximation is acceptable for small variations in parameters that do not directly affect the spatial distribution of amplitude. Sensor tilt would for example change the shape of the signal amplitude response as function of coordinates. A tilt of five degrees will lead to an error of the order of $10 \%$ around the area of maximum amplitude which may not be acceptable.

\subsubsection{Results using model generated POD data}

The selection of the input parameter distributions has a large impact on the modelled result. The experimental system used here is well controlled and allows a well defined input to be used for the simulations. The distribution of the contact parameter $\zeta$ is selected to be normally distributed with $\mu_{\zeta}=0.45$ and $\sigma_{\zeta}=0.05$, based on the result of Fig. 6. The lift-off is set as normally distributed with $\mu_{L}=0.1 \mathrm{~mm}$ and $\sigma_{L}=1 \mu \mathrm{m}$. The crack position is uniformly distributed between $\pm L_{\text {scan }} / 2$ relative the sensor scan 




Fig. 8. Error as a function of sensor coordinates. White areas do not represent possibilities for maximum amplitude during straight scans.

and the orientation is assumed to be normally distributed around zero with $\sigma_{\text {orient }}=2.5^{\circ}$. Using this data and generating 54 computed signal responses from nine different crack sizes gives the resulting data and POD curves shown in Fig. 9. A scan index distance $L_{\text {scan }}$ of $0.5 \mathrm{~mm}$ and $1.0 \mathrm{~mm}$ is compared with experimentally obtained data. The result in the case of $L_{\text {scan }}=0.5 \mathrm{~mm}$ gives a constant variance $v_{M L}=0.0478$ and the values of $a_{90}$ and $a_{90 / 95}$ are $0.8225 \mathrm{~mm}$ and $0.8556 \mathrm{~mm}$ respectively. For $L_{\text {scan }}=1.0$ $\mathrm{mm}$ the results are $v_{M L}=0.0826, a_{90}=0.9382 \mathrm{~mm}$ and $a_{90 / 95}=0.9888 \mathrm{~mm}$.

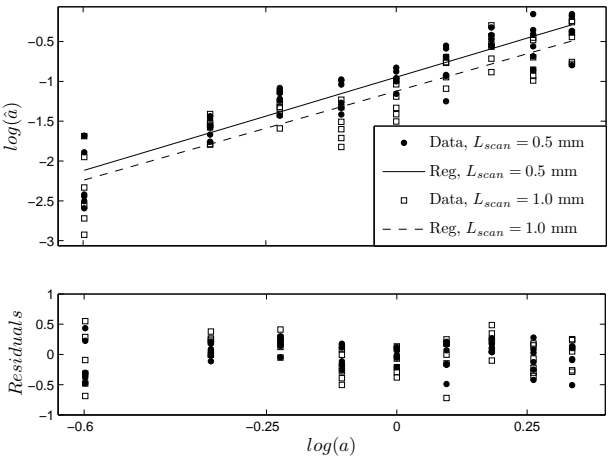

(a) Model generated $\hat{a}$ vs $a$ data.

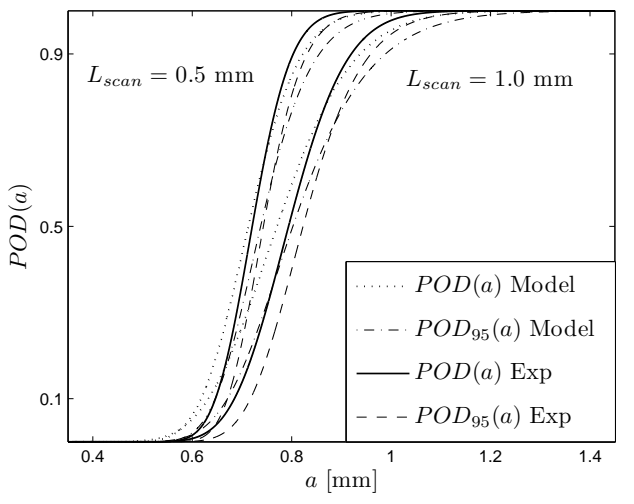

(b) Estimated model based POD curve compared with the experimental result.

Fig. 9. Results of model based POD.

The result is depending on the distribution of the crack orientation. Table 4 shows how the values of $a_{90}$ and $a_{90 / 95}$ change with the selected distribution parameters, holding the others constant. It can be concluded from the table that the small standard deviation of the crack orientation distribution keeps 
a conservative POD estimation. The table shows also a good agreement with the experimental data in Fig. 4. The results will be somewhat different between individual runs due to the statistical nature of the result and the limited number of generated data, which here is 54 points at nine crack sizes as presented in Fig. 9. The model is not conservative for small cracks, which can be concluded as the variations around the regression line are similar to the experimental data. This is due to the model description which overestimates the signal response from small cracks.

Table 4. Influence from crack orientation on POD.

\begin{tabular}{l|l|l|l|l} 
Source & $\mathbf{L}_{\text {scan }}$ & $\boldsymbol{\sigma}_{\text {orient }}$ & $\boldsymbol{a}_{\mathbf{9 0}}$ & $\boldsymbol{a}_{\mathbf{9 0} / \mathbf{9 5}}$ \\
\hline Model & 0.5 & $0^{\circ}$ & 0.8152 & 0.8458 \\
& 0.5 & $2.5^{\circ}$ & 0.8226 & 0.8556 \\
& 0.5 & $5^{\circ}$ & 0.8057 & 0.8361 \\
& 0.5 & $30^{\circ}$ & 0.806 & 0.8373 \\
\hline Exp. & 0.5 & $1-5^{\circ}$ & 0.8031 & 0.8313 \\
\hline Model & 1.0 & $0^{\circ}$ & 0.9528 & 0.9963 \\
& 1.0 & $2.5^{\circ}$ & 0.9382 & 0.9888 \\
& 1.0 & $5^{\circ}$ & 0.896 & 0.9334 \\
& 1.0 & $30^{\circ}$ & 0.8913 & 0.934 \\
\hline Exp. & 1.0 & $1-5^{\circ}$ & 0.9199 & 0.9594 \\
\hline
\end{tabular}

\subsection{Approach 2: Point estimation for non-parametric POD curve}

The POD function of crack size $a$ can be formulated as

$$
\operatorname{POD}(a)=\int_{\hat{a}_{d e c}}^{\infty} g_{a}(x) d x
$$

where $a$ is the crack size parameter, $\hat{a}_{d e c}$ is the decision threshold and $g_{a}$ is a function describing the signal response distribution at $a$. The standard approach of estimating $P O D(a)$ assumes that there is a linear relation between the signal response $\hat{a}$ and the crack size $a$ with the function $g_{a}$ as a normal distribution with mean at the regression line and constant variance for all crack sizes $a$. These restrictions must be fulfilled in order to build the parametric POD curve. However, a model of the eddy current procedure can estimate the signal distribution at discrete crack sizes. This distribution will not in general be the same for each crack size and not normally distributed. The standard approach for $\hat{a}$ versus $a$ POD uses information of the response variation for all cracks to estimate the constant variance. The model approach in this case will instead be a point estimate on the POD curve at the selected crack size. Using several crack sizes will give a resulting non-parametric POD curve. It is common to use the lower 95\% confidence limit to estimate a POD that is conservative in its nature. This is not possible with a model based POD estimation using the non-parametric approach. This means that there is also a need to have another way to generate a conservative estimation of POD for a model based result. Here, this 
is proposed to be included by a conservative fatigue crack model described above. Thus, if the predicted signal amplitude is lower than the experimentally obtained values, a conservative POD may be obtained if variations in procedure parameters are adequate.

\subsubsection{Results using point probability}

A simplified meta-model for each crack size is built, including only the contact parameter variation, ignoring the lift-off variation. A more general meta-model could include many parameters, also crack size. The model used here is constructed from the result of uniformly distributed values of $\zeta$ at each crack size and a linear response curve is considered for each crack size. A number of 5000 computations for each crack size $a$ are evaluated. The parameters are set up with normally distributed crack orientation with $\left(\mu_{\text {orient }}, \sigma_{\text {orient }}\right)=(0,2.5)^{\circ}$, scan position relative the centre of the defects that is uniform on $\left[-L_{\text {scan }} / 2, L_{\text {scan }} / 2\right]$ and a crack contact represented by a normally distributed parameter $\zeta$ with $\left(\mu_{\zeta}, \sigma_{\zeta}\right)=(0.45,0.05)$. The result is presented in Fig. 10 together with the experimental result from section 4.



Fig. 10. Simplified model based POD compared with experimentally obtained curve and its lower $95 \%$ confidence limit.

Linear interpolation is used between the simulated points on the POD curve of Fig. 10. The model based POD curve does not follow a cumulative normal distribution function, as is the case of the experimental data. The agreement is good and the conservative selection of crack model and its variation is capturing the major effects on POD from the crack characteristics. The impact from the omitted lift-off is small since this parameter has a limited variation on the captured crack signal amplitude.

The impact from the distribution of the fatigue crack orientation is presented in Fig. 11. The two different 
scan index distances considered here are shown. It can be concluded that the use of a small variance of the crack orientation around a parallel crack alignment is conservative up to a point where the scan index distance is large. At that point the capability is reduced as the orientation of the crack is close to being perpendicular to the scan direction. This is due to the signal characteristics of small defects giving signal peaks as the sensor is positioned at the corners of the crack where the induced currents are interrupted most effectively. The crack is thus detected with a reduced probability if the scan line is positioned over the centre of a perpendicularly oriented crack and the scan index distance is large.
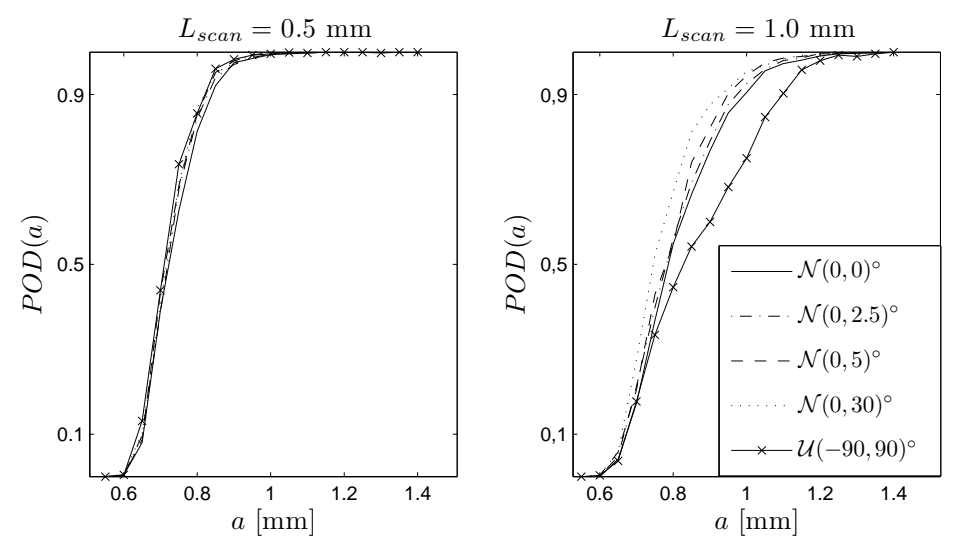

Fig. 11. Distribution of crack orientation considered for two different scan index distances.

Scan directions that are parallel and perpendicular to the crack orientation are considered when estimating the change in POD as the scan index distance, $L_{\text {scan }}$ is changed. It is relevant to consider these two limits of crack orientations as it has been concluded that they represent the most conservative case for the POD estimate. The result as the scan index distance is changed in four steps is shown in Fig. 12.
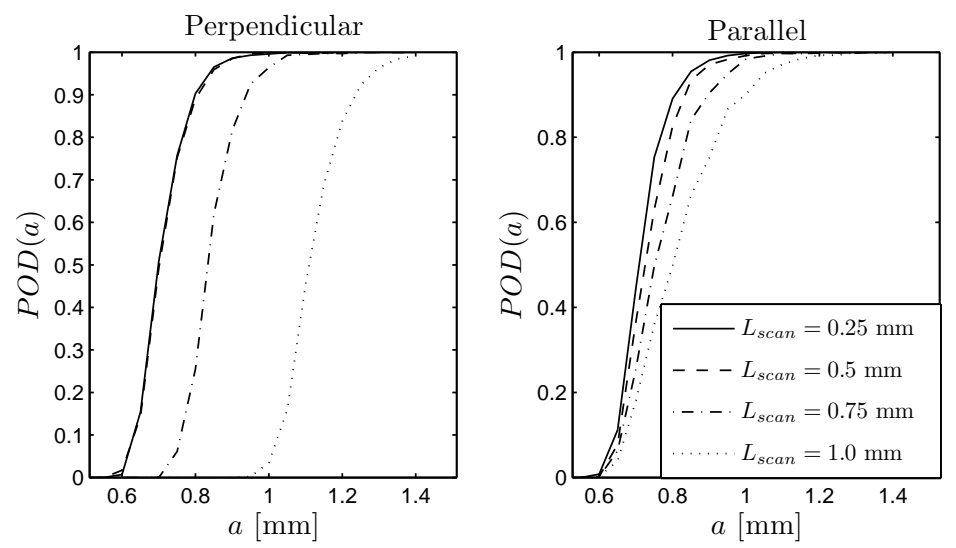

Fig. 12. Change in POD as scan index distance is increased as the crack is aligned parallel respectively perpendicular to the scan orientation. 
Fig. 12 shows the importance of the scan index distance and gives an indication of what may be sufficient to use in order to get an effective inspection procedure.

\section{Discussion}

Using only a model based POD instead of an experimental assessment may still lie ahead of us. This work has considered a simplified and well controlled procedure. Critical variables of the model have been identified from the experimental POD and must be considered in any changes of the procedure. If, for example, the material would be different, it would be necessary to establish a new crack model and to carefully verify this in experiments. The model based approach does also require a detailed knowledge of the procedure as parameter distributions must be estimated and justified in order to achieve a relevant result. The modelled result relies on these inputs and they can only be tested as long as there are experimental results that can be used for validation purposes. The model based approach may, however, be used efficiently if a few experimental POD curves are produced and the model are used for studies of parameter changes of the procedure, concluding trends and important changes due to such variations. Modelling tools are vital for the POD estimation of procedures applied to complex shaped parts due to the complexity and cost of the experimental assessments.

One of the essential features in model based POD assessments considering automated eddy current procedures is the defect description. In this work a model is developed that predict the signal amplitude characteristics for the cracks used in the experiments. This model may have deviations if the interaction between the crack and the magnetic fields emitted from the probe is altered. Changes in applied frequency of the field may thus need to be accompanied by another crack model. Work is thus needed on how to model the natural crack and how the characteristics of this best should be determined.

\subsection{Probability of false alarms}

The selection of crack model results in a POD estimation that is conservative in this case. However, in the point estimation approach, as sources of noise from lift-off and bulk material characteristics are ignored, the probability of false alarm (PFA) is always 0 . This can be viewed as the signal response without any crack in the model. If a lowered decision threshold is used or a larger variation in lift-off can be expected it might be highly relevant to also characterize the PFA. Using an assumed lift-off variation that is normally distributed with $\sigma_{L}=2.5 \mu \mathrm{m}$ gives the distribution of noise and defect according to Fig. 13. The lift-off response is calculated from the response of a shallow defect of dimensions $2 \times 2 \mathrm{~mm}$ 
centred underneath the sensor, in order to represent the response of the surface variation. The signal distribution is representing a crack of size $0.85 \mathrm{~mm}$. The figure shows the large impact from the still quite small lift-off variation. This is a reason why the impedance component separated in phase by $90^{\circ}$ from the lift-off direction is often used as signal response parameter in eddy current procedures, even if automated.

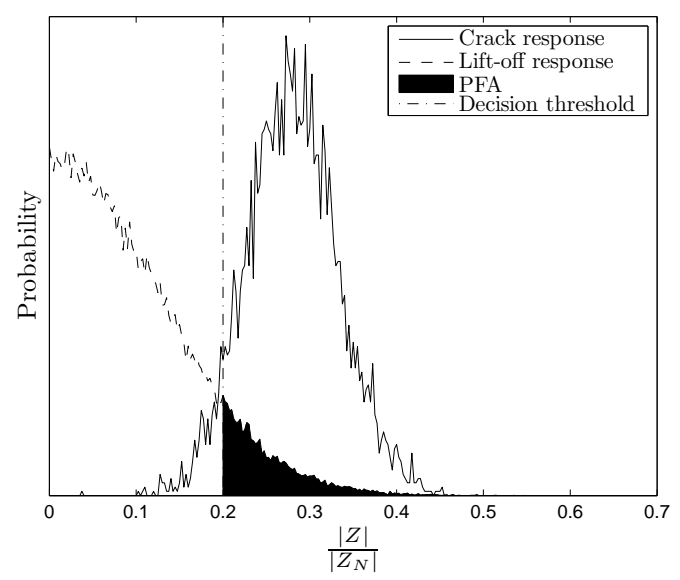

Fig. 13. Signal response distributions from $0.85 \mathrm{~mm}$ long crack and lift-off respectively.

\subsection{Transfer function approach}

The transfer function approach may represent the first choice of selection using knowledge from models in the context of POD $[16,17]$. The use of experimental input is important in order to build confidence of the result but may be approximate and the final steps can be justified by a validated mathematical model. The methods applied using the transfer function approach may be tested using the presented data within this work, even if not representing the most efficient use of models. A typical use of this method would be to study the effect from probe position on a complex geometry compared to the base line POD which is estimated on flat surfaces. Assume first that the base line POD curve is generated experimentally using $L_{\text {scan }}=0.5 \mathrm{~mm}$. The linear regression is here described as $\log (\hat{a})=\hat{\beta}_{0}+\hat{\beta}_{1} \log (a)+\hat{\epsilon}_{\beta}$. The random error term, $\hat{\epsilon}_{\beta}$ is normally distributed with zero in mean and standard deviation estimated from the data as $\hat{\sigma}_{\beta}$. The model results can then be used to estimate the POD change as $L_{\text {scan }}$ is increased to 1 mm. Using the parametric POD model is leading to a change in the estimated regression parameters from $\left(\hat{\xi}_{0}^{\left(L_{0.5}\right)}, \hat{\xi}_{1}^{\left(L_{0.5}\right)}, \hat{\epsilon}_{\xi}^{\left(L_{0.5}\right)}\right)$ to $\left(\hat{\xi}_{0}^{\left(L_{1}\right)}, \hat{\xi}_{1}^{\left(L_{1}\right)}, \hat{\epsilon}_{\xi}^{\left(L_{1}\right)}\right)$ according to the results from the FEM computations, where $\left(L_{0.5}\right)$ and $\left(L_{1}\right)$ denotes the procedure using $L_{\text {scan }}$ of 0.5 and $1 \mathrm{~mm}$ respectively. The new POD curve is estimated using the experimentally obtained base line result, together with the information from the change between the modelled results. The model can include variations in contact, lift-off, orientation 
and position. It is, however, more realistic to only include the variable that relates to the scan index distance, $L_{\text {scan }}$. The parameters $\hat{\xi}_{0}$ and $\hat{\xi}_{1}$ in those two model based cases are used to compute the change on the experimental regression line. The difference in standard deviation of the random error term, $\hat{\sigma}_{\Delta \xi}=\hat{\sigma}_{\xi^{\left(L_{1}\right)}-\xi^{\left(L_{0.5}\right)}}=\sqrt{\hat{\sigma}_{\xi^{\left(L_{1}\right)}}^{2}-\hat{\sigma}_{\xi^{\left(L_{0.5}\right)}}^{2}}$ is also added to the experimental data. This treatment holds as it is assumed that the random error term is normally distributed. The new transferred POD curve is now based on the relation

$$
\begin{aligned}
\log \left(\hat{a}^{\left(L_{1}\right)}\right) & =\log \left(\left[\frac{\exp \left[\hat{\xi}_{0}^{\left(\mathrm{L}_{1}\right)}+\hat{\xi}_{1}^{\left(\mathrm{L}_{1}\right)} \log (\mathrm{a})\right]}{\exp \left[\hat{\xi}_{0}^{\left(\mathrm{L}_{0.5}\right)}+\hat{\xi}_{1}^{\left(\mathrm{L}_{0.5}\right)} \log (\mathrm{a})\right]}\right] \hat{a}^{\left(L_{0.5}\right)}\right)+\hat{\epsilon}_{\beta\left(L_{0.5}\right)+\Delta \xi} \\
& =\hat{\beta}_{0}^{\left(L_{1}\right)}+\hat{\beta}_{1}^{\left(L_{1}\right)} \log (a)+\hat{\epsilon}_{\beta}^{\left(L_{1}\right)}
\end{aligned}
$$

The simple treatment of data relies on the parametric POD model which is used both for the experimental and synthetic data. The new set of regression parameters are used to calculate the new POD curve.

Another treatment of model results must be used if information from a non-parametric model based approach is to be used. In that case the change in POD must be added to the resulting experimentally based POD curve. The change in the model based curve $\triangle P O D^{(M)}(a)=P O D^{(M)}\left(a ; L_{1}\right)-P O D^{(M)}\left(a ; L_{0.5}\right)$ is added as

$$
P O D\left(a ; L_{1}\right)=P O D^{(E)}\left(a ; L_{0.5}\right)+\Delta P O D^{(M)}(a)
$$

where ${ }^{(E)}$ and ${ }^{(M)}$ denote if the function is established from experimental or modelled data, respectively. The new POD curve will in this case not follow a cumulative normal distribution function.

There are a few choices that are worth to be discussed. The following notations are used for the presentation of results in Fig. 14 where $\triangle P O D$ is calculated using the finite element model.

- $\triangle P O D_{1}$ : Parametric POD model is used and variations in contact, lift-off, crack orientation and crack position included. $\triangle P O D$ is calculated according to Eq. (4).

- $\triangle P O D_{2}$ : Parametric POD model is used. Nominal values for contact, lift-off and orientation are applied. Position is described with variations and $\triangle P O D$ is calculated according to Eq. (4).

- $\triangle P O D_{3}$ : Non-parametric POD model is used, variations in contact, lift-off, crack orientation and crack position included. $\triangle P O D$ is calculated according to Eq. (5). 
- $\triangle P O D_{4}$ : Non-parametric POD model is used, nominal values for contact, lift-off and crack orientation are applied. Position is described as a distribution. $\triangle P O D$ is calculated according to Eq. (5).

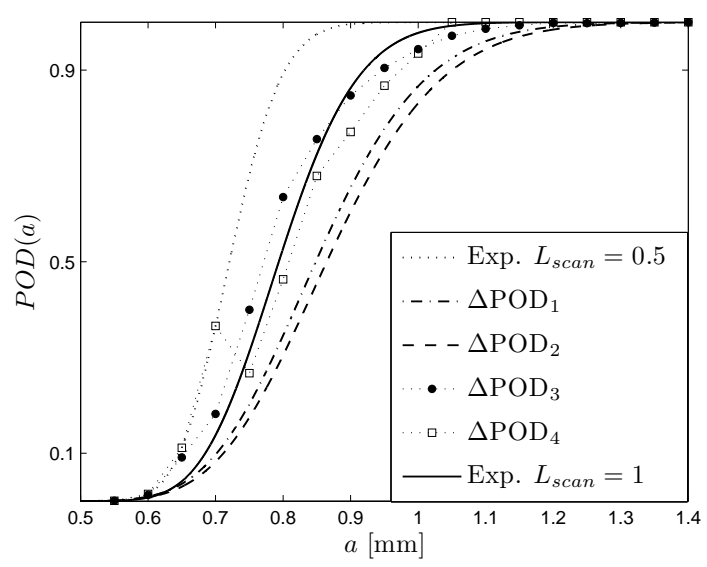

Fig. 14. Results from $\triangle P O D$ using transfer functions compared to the experimental parametric POD.

The results are compared to the experimentally generated POD curve using $L_{\text {scan }}=1 \mathrm{~mm}$ in Fig. 14 . All confidence bounds are left out from the figure for clarity. It must be pointed out that the confidence bound cannot be added using the delta POD approach with non-parametric model results. The lower confidence from the base line POD curve can be shifted with $\triangle P O D$ as a first approximation. The results from $\triangle P O D_{2,4}$ represents the most feasible option for real use as these only include variation in the variable which is left out of consideration in the new POD. In that case it is assumed that the POD change from the $L_{\text {scan }}$ parameter is uncorrelated with variations in the other parameters. This is not the case which is clear from the difference between $\triangle P O D_{1}$ and $\triangle P O D_{2}$ and also between $\triangle P O D_{3}$ and $\triangle P O D_{4}$. This is mainly due to the treatment of the orientation of the crack. The difference between the delta POD using the parametric and non-parametric approach is related to the crack model. This model has good agreement at the crack sizes relevant in the area where the POD curve is changing rapidly. The parametric approach is considering a larger range of defects and is underestimating signal responses for larger cracks. The conservative treatment is transferred to the POD curve using the parametric approach. The resulting curve using $\triangle P O D_{4}$ is showing an erroneous behaviour as the POD curve is decreasing for a certain range of crack sizes. This is not physically correct but originates from the fact that the non-parametric POD curve has a different shape. This curve must thus be ignored for crack sizes less than $0.75 \mathrm{~mm}$. 


\section{Conclusions}

A model based POD is a useful tool in the study of automated eddy current procedures. Method capability trends due to changes in parameter distributions can be studied and used when considering the final procedure for an automated inspection. The model based POD may be considered both by the study of synthetic data treated analogously to experimental data and by the use of estimations using signal response distributions. Both methods show good agreement with the experimental data produced within this work. The first approach must be evaluated in the sense that the data fulfils the criteria to be handled with the classical POD model. The signal response may for example deviate significantly from the linear model which calls for other statistical treatment of the data. The second approach used here is directly representing the POD estimation and needs no additional treatment to produce the resulting curve. A conservative result is important for a model based POD. This can be assured by applying relevant input parameters to the model. The $\triangle P O D$ approach is a vital tool in this and is shown here to be less sensitive to the description of crack and input variations in the model. The experimentally obtained base line curve is also building confidence in the result which promotes the use of model based POD using the transfer function approach to arrive at a conservative result.

The fatigue crack model is essential. The model can be constructed aided by signal responses evaluated from a small number of experiments. The model is selected here in order to be conservative, not overestimating the signal amplitude or the spatial distribution. It is important to evaluate the crack model in the entire range of defects used to estimate the POD curve. This has also an impact using transfer functions which is shown to be possible using information gained from models. The work presented is pointing out important future challenges in modelling of the natural crack and determination of the distributions of crack as well as procedure parameters. The final result is strongly dependent on the judgement of these parameters.

\section{Acknowledgement}

This work is carried out within the frame of a European collaboration project, PICASSO. The support from GKN Aerospace Engine Systems in this research as well as the funding from the European Commission within the FP7 programme is greatly acknowledged. 


\section{References}

[1] Nondestructive evaluation system reliability assessment. USA Department of Defense Handbook MIL-HDBK-1823. 2009.

[2] R.E. Beissner and J.S. Graves III. "Computer Modeling of Eddy Current Probability of Crack Detection". Review of progress in quantitative nondestructive evaluation. Ed. by D.O. Thompson and D.E. Chimenti. Vol. 9A. Plenum Press, 1990, pp. 885-891.

[3] N. Nakagawa and E. Beissner. "Probability of Tight Crack Detection via Eddy-Current Inspection". Review of progress in quantitative nondestructive evaluation. Ed. by D.O. Thompson and D.E. Chimenti. Vol. 9A. Plenum Press, 1990, pp. 893-899.

[4] N. Nakagawa, M.W. Kubovich, and J.C. Moulder. "Modeling Inspectability for an Automated Eddy Current Measurement System". Review of progress in quantitative nondestructive evaluation. Ed. by D.O. Thompson and D.E. Chimenti. Vol. 9A. Plenum Press, 1990, pp. 1065-1072.

[5] S.N. Rajesh, L. Udpa, and S.S. Udpa. "Numerical Model Based Approach for Estimating POD in NDE Applications". IEEE Transactions on Magnetics 29 (2 1993), pp. 1857-1858.

[6] J.S. Knopp et al. "Inverstigation of a Model-Assisted Approach to Probability of Detection Evaluation". AIP Conf. Proc. Review of Progress in Quantitative Nondestructive Evaluation (Portland, Oregon (USA)). Vol. 894. 2007, pp. 1775-1782.

[7] N. Dominguez and F. Jenson. Simulation Assisted POD of a high Frequency Eddy Current Inspection Procedure. 10th European Conference on Non-Destructive Testing. 2010.

[8] A. Rosell and G. Persson. "Finite element modelling of closed cracks in eddy current testing". International Journal of Fatigue 41 (2012), pp. 30-38.

[9] A.P. Berens. "NDE reliability data analysis". Nondestructive Evaluation and Quality Control. Vol. 17. ASM Metals Handbook. ASM International, 1989, pp. 689-701.

[10] W.D. Rummel. "Recommended Practice for Demonstration of Nondestructive Reliability on Aircraft Parts". Materials Evaluation 40 (1982), pp. 922-932.

[11] Y. Zhang, F. Luo, and H. Sun. Impedance Evaluation of a Probe-Coil's Lift-off and Tilt Effect in Eddy-Current Nondestructive Inspection by 3D Finite Element Modeling. Proceedings of the 17th World Conference on Nondestructive Testing. 2008.

[12] C. Dodd and W. Deeds. "Analytical solutions to eddy-current probe-coil problems". Journal of Applied Physics 39.6 (1968), pp. 2829-2838. 
[13] B.A. Auld, F. Muennemann, and D.K. Winslow. "Eddy current probe response to open and closed surface flaws". Journal of Nondestructive Evaluation 2.1 (1981), pp. 1-21.

[14] R.E. Beissner. "Slots vs. Cracks in Eddy Current NDE". Journal of Nondestructive Evaluation 13.4 (1994), pp. 175-183.

[15] J.R. Bowler. "Eddy-current interaction with an ideal crack. I. The forward problem". J. Appl. Phys. 75.12 (1994), pp. 8128-8137.

[16] R.B. Thompson. "Using physical models of the testing process in the determination of Probability of Detection". Materials Evaluation 59.7 (2001), pp. 861-865.

[17] R.B. Thompson. "A Unified Approach to the Model-Assisted Determination of Probability of Detection". AIP Conf. Proc. Review of Progress in Quantitative Nondestructive Evaluation (Golden, Colorado). 975. 2007, pp. 1685-1692. 